\title{
Erwhi Hedgehog: A New Learning Platform for Mobile Robotics
}

\author{
Giovanni di Dio Bruno
}

\begin{abstract}
Erwhi Hedgehog is one of the smallest mobile robots. It enables mapping and vision analysis, and also displays machine learning features. Behaving like a small, curious animal, eager to explore the surroundings, the robot can be used to test navigation, mapping and localization algorithms, thus allowing the prototyping of new hardware and software for robotics. This application is particularly handy for educational robotics, at both high school and university level. On the one hand, the project is fully open source and open hardware under MIT license and available on Github, so everyone can build his/her own Erwhi Hedgehog robot with the aid of a step-by-step guide. On the other hand, students with more advanced knowledge can use it as a prototyping platform for developing new software programs and features. Erwhi uses Intel RealSense, AAEON UP Squared and Myriad X VPU technologies, with software based on the Robotic Operating System (ROS), and implements SLAM algorithms, such as RTAB-Map. The machine learning aspect is based on the OpenVINO framework and a dedicated ROS wrapper was used. The software package includes all the programs needed to create a Gazebo simulation. In terms of hardware, motor control is based on an STM32 microcontroller and the Arduino software, and the robot works on the differential drive unicycle model. Finally, Erwhi is compatible with AWS RoboMaker tools.
\end{abstract}

Keywords Erwhi $\cdot$ ROS $\cdot$ Robotics $\cdot$ Machine learning $\cdot$ SLAM $\cdot$ Vision analysis

\section{Introduction}

Building a robot is an opportunity for students to learn several aspects of technology, from the design of functional hardware to the development of sophisticated software. Simultaneous mapping, vision analysis and machine learning are necessary features for several types of robots, and have countless applications. Students can currently build robots with these features using Lego kits or Robotis TurtleBot 3. While they

\footnotetext{
G. D. Bruno $(\bowtie)$

Ass. Officine Robotiche, Rome, Italy

URL: http://gbr1.github.io 
Fig. 1 Front and rear view of Erwhi Hedgehog robot

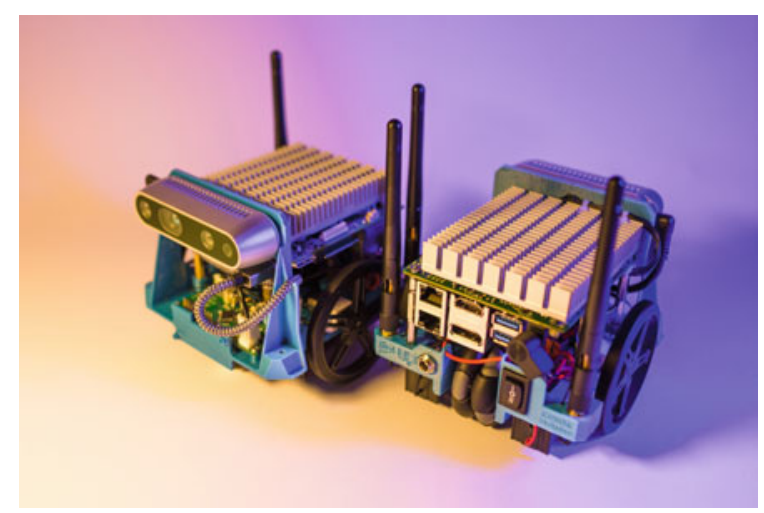

are both powerful tools, they also have their limitations. When it comes to building hardware, Lego kits provide flexibility, but they mostly rely on block programming, which can be limiting when developing more complex software. TurtleBots is flexible for both hardware and software, but requires expensive upgrading for special applications, such as object recognition.

Erwhi Hedgehog is one of the smallest mobile robots currently available. It allows simultaneous mapping, vision analysis and machine learning, and is completely open source and open hardware [1], so anyone can build or customize his/her own robot (Fig. 1). The robot can behave like a small, curious animal, exploring its surroundings, and can be used to test navigation, mapping, and localization algorithms. The aim of the entire project is to accelerate and simplify the development of robotics for researchers, educators, students and professionals, by providing a platform for prototyping new robotics hardware and software, and learning by doing.

\section{Description of the Robot}

\subsection{Electronics}

The main core of Erwhi Hedgehog's electronics (Fig. 2) is an UP Squared Atom x7-E3950 with 4 GB of DDR4 RAM and 64 GB of EMMC non-volatile memory. Vision is provided by an Intel® RealSense D435 depth camera. Machine learning runs on an AI Core X, Movidius ${ }^{\mathrm{TM}}$ Myriad $^{\mathrm{TM}}$ X VPU on a PCIe board. Hard realtime controls are implemented on a custom carrier board called Sengi, and based on an STM32F103C8T6 micro controller. The main board is connected to Sengi via USB1.1. The micro controller is connected to a power motor driver and motor encoders. The carrier board includes an absolute Inertial Motion Unit BNO055 that is connected to the main core via a USB-serial adapter. Power is supplied by two 


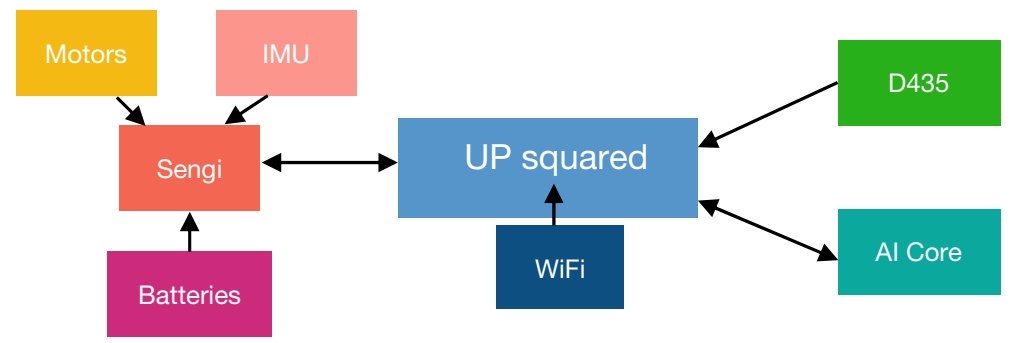

Fig. 2 Simplified diagram of the electronics of Erwhi Hedgehog

18,650 Li-Ion cells in series, with about $8.4 \mathrm{~V}$ at maximum charge. Power flows through a DC/DC step down converter which handles an output of $5.1 \mathrm{~V}$ and $8 \mathrm{~A}$ for a continuous load. The robot communicates over WiFi at $2.4 / 5 \mathrm{GHz}$, and Bluetooth.

\subsection{Mechanical Parts}

Mechanical parts are designed to be 3D printed using classical methodologies, such as the commonly used fused filament fabrication.

Erwhi Hedgehog is actuated by two DC gearmotors, with the robot moving on two casters, a ball caster at the front and an omniwheel at the rear.

\subsection{Software}

Erwhi Hedgehog runs on Ubuntu 16.04 LTS and ROS [2] Kinetic Kame (Fig. 3). The robot's mathematical model is implemented via the standard ROS control package [3], making the code very easy to adapt to any robot, simply by adjusting a few parameters. The joints are controlled by Sengi firmware, using the Arduino language and STM32duino libraries [4]. Its movements are based on the standard ROS navigation

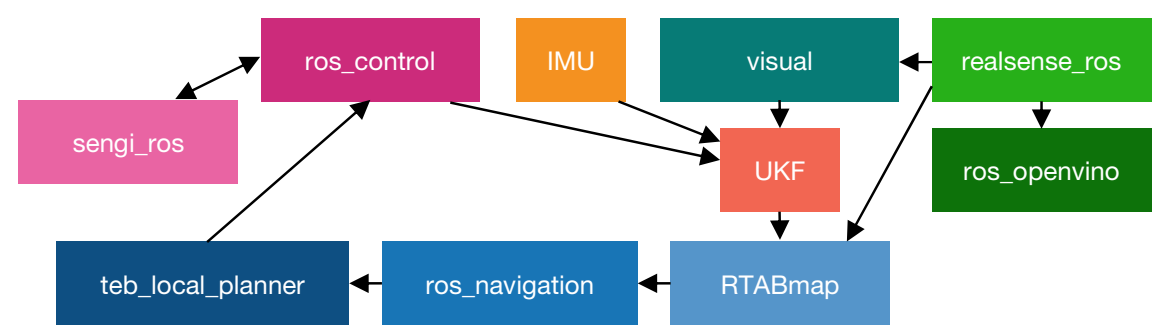

Fig. 3 Simplified diagram of the ROS architecture used in Erwhi Hedgehog 
stack [5] and a timed-elastic-band (TEB) planner [6]. Simultaneous localization and mapping (SLAM) works on RTAB-Map [7] and robot localization is provided by the unscented Kalman filter (UKF) [8], which combines data from the visual odometry, the wheel odometry and the IMU. Erwhi Hedgehog is also implemented in Gazebo Simulator, which means that the robot can be simulated in different environments and conditions. The software is fully compatible with AWS RoboMaker. Vision tasks are implemented through the Intel OpenVINO framework.

\section{Learning and Prototyping by Doing}

\subsection{Erwhi Hedgehog as a Teaching Tool}

As a very recent project, Erwhi Hedgehog has not been used in schools yet. However, its small size has made it very popular at mass events, such as Maker Faires, where it facilitates the display of UGV (unmanned ground vehicle) technologies and functionalities to a wide audience, including families with young children. Erwhi Hedgehog has also been tested and used in ROS and advanced mobile robotics workshops as a benchmark platform for studying differences in various planners and SLAM algorithms. Hands-on testing of different planners and SLAM algorithms have proved to be an effective way of learning their distinctive features and weaknesses, such as their computational complexity. It also provides an opportunity for workshop attendees, mainly university students and freelancers, to use these platforms.

\subsection{Learning by Modifying Hardware}

Users can also add to or edit parts of Erwhi Hedgehog's hardware through the different sensors and actuators. For example, the robot can easily be connected to an Arduino board to make it usable with all Arduino-compatible sensors and actuators. This makes way for infinite application possibilities.

\subsection{Learning New Technologies}

The robot helps to test vision algorithms based on deep learning, such as object recognition. These algorithms can be tested directly, which makes it easier to understand how they work and how they can be used in robotics. Furthermore, AWS RoboMaker facilitates the creation and simulation of custom and complex software in the cloud, 
which eliminates the need for powerful local computers. In addition, it can also be used to deploy custom applications to Erwhi Hedgehog over the Internet of Things (IoT), making it is possible to work on a remote physical robot.

\section{Conclusions}

Erwhi Hedgehog is a good platform for gaining hands-on knowledge and experience of mobile robotics from the hardware to the software. It also represents the current status of mobile robotics, based on the latest industrial technologies. The platform should not be considered merely a standalone robot, but rather a reference design that students and educators can use to advance their projects, e.g., by adding computer vision to an existing project using only Erwhi Hedgehog software on different hardware.

The current focus of development in the project is updating and simplifying the robot.

\section{References}

1. Erwhi Hedgehog main Github repository. https://github.com/gbr1/erwhi-hedgehog (2019). Latest commit $930 \mathrm{fb} 8 \mathrm{c}$ on 21 August 2019

2. Quigley, M., Conley, K., Gerkey, B., Faust, J., Foote, T., Leibs, J., Wheeler, R., Ng, A.: ROS: an open-source robot operating system. ICRA workshop on open source software, $\mathbf{3}$ (2009)

3. Chitta, S., Marder-Eppstein, E., Meeussen, W., Pradeep, V., Tsouroukdissian, A., Bohren, J., Coleman, D., Magyar, B., Raiola, G., Lüdtke, M., Perdomo, E.: ros_control: a generic and simple control framework for ROS. J. Open Source Softw. 2, 456 (2017). https://doi.org/10.21105/joss. 00456

4. STM32duino Arduino core Github repository. https://github.com/stm32duino/Arduino_Core_ STM32 (2019). Latest commit 964576b 26 September 2019

5. Marder-Eppstein E., Berger, E., Foote, T., Gerkey, B., Konolige, K.: The office marathon: robust navigation in an indoor office environment. In: 2010 IEEE International Conference on Robotics and Automation, Anchorage, AK, pp. 300-307 (2010). https://doi.org/10.1109/ROBOT.2010. 5509725

6. Teb_local_planner ROS package documentation. http://wiki.ros.org/teb_local_planner (2019). Latest access 6 October 2019

7. Labbé, M., Michaud, F.: RTAB-map as an open-source lidar and visual SLAM library for largescale and long-term online operation. J. Field Robot. 36(2), 416-446 (2019)

8. Robot localization ROS package documentation. http://docs.ros.org/melodic/api/robot_localizat ion/html/index.html (2019). Latest access 6 October 2019 
Open Access This chapter is licensed under the terms of the Creative Commons Attribution 4.0 International License (http://creativecommons.org/licenses/by/4.0/), which permits use, sharing, adaptation, distribution and reproduction in any medium or format, as long as you give appropriate credit to the original author(s) and the source, provide a link to the Creative Commons license and indicate if changes were made.

The images or other third party material in this chapter are included in the chapter's Creative Commons license, unless indicated otherwise in a credit line to the material. If material is not included in the chapter's Creative Commons license and your intended use is not permitted by statutory regulation or exceeds the permitted use, you will need to obtain permission directly from the copyright holder.

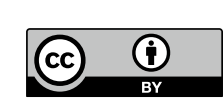

